\title{
Detection of vaneless diffuser rotating stall by means of dynamic pressure sensors and acoustic measurements
}

\author{
Luca Romani $^{1}$, Lorenzo Bosi ${ }^{1}$, Alberto Baroni ${ }^{1}$, Lorenzo Toni ${ }^{2}$, Davide Biliotti ${ }^{2}$, Giovanni \\ Ferrara $^{1}$, and Alessandro Bianchini ${ }^{1, *}$ \\ ${ }^{1}$ Department of Industrial Engineering, Università degli Studi di Firenze, 50139 Firenze, Italy - \\ *alessandro.bianchini@unifi.it \\ ${ }^{2}$ Baker Hughes, 50127 Firenze, Italy
}

\begin{abstract}
An accurate estimation of rotating stall is one of the key technologies for high-pressure centrifugal compressors. Several techniques have been proposed to detect the stall onset; inter alia, few dynamic pressure probes have been shown to not only properly detect the phenomenon, but also reconstruct the stall characteristics after an ensemble averaging approach. The massive use of this technique in the field is, however, not a common practice yet. In the present study, the use of dynamic pressure probes has been combined with that of an environmental microphone to evaluate the prospects of this latter for a possible stall onset detection. To this end, experimental tests have been carried out in the experimental test rig of the Department of Industrial Engineering (DIEF) of Università degli Studi di Firenze. Results show that the microphone was able to distinguish the onset of rotating stall accurately and promptly, even though - differently from dynamic pressure sensors - it does not provide sufficient information to determine the characteristics of the stall pattern. On this basis, the use of acoustic measurements could find room for automatic detection of rotating stall onset.
\end{abstract}

\section{Introduction}

Centrifugal compressors do represent a key technology in the present energy scenario. Parallel to conventional applications in the oil and gas sector, in fact they are finding new room for application also in the developing economy of hydrogen, where high-efficiency compression is needed for both storage and distribution [1]. The intrinsic variability of these applications is posing even more focus on a research trend already present in gas application, i.e. the increase of the rangeability of the stages towards lower minimum flow limits. To this end, enhancing the comprehension of effects those aerodynamic phenomena that precede surge is of capital importance [2]. In particular, since the use of vaneless diffusers is often suggested in all those cases where a good rangeability is needed, vaneless diffuser rotating stall (VDRS) has attracted the attention of researchers for many years $[3,4]$. This aerodynamic phenomenon is characterized by the creation inside the diffuser of a non-axisymmetric pressure pattern, which rotates almost rigidly with a subsynchronous 
frequency generally lower than $20-30 \%$ of the revolution frequency [5,6]. This pattern is usually made of a small number (1-4) of stall cells or lobes [7]. The effect of this aerodynamic unbalance is the creation of a rotating force [8,9], which acts as a destabilizing external load on the rotordynamics of the compressor [10], possibly preventing the machine to operate in or beyond that operating condition. As a consequence, much attention is paid at developing reliable techniques to early detect the onset of rotating stall.

To this end, a variety of proposal can be found, ranging from displacement probes mounted on the shaft [11] to magnetic bearings with vibration control systems [12] or piping vibration systems [13]. The two major approaches to tackle rotating stall detection are based, however, on dynamic pressure (e.g. [14]) or acoustic measurements (e.g. [15]). Both approaches have been applied up to now mainly in research applications, due to the issues related to the operation of sensors in a harsh real environment. However, nice prospects have been shown by each of the two methods. More specifically, the use of dynamic pressure sensors was shown to be able not only to reconstruct the stall pattern [16], but also to possibly represent a valuable tool for determining an automatic onset threshold [2]. On the other hand, acoustic measurements have been proposed as a valuable alternative [17], being less intrusive than flush-mounted pressure probes.

In the present study, an experimental analysis is presented to compare the two methods. A real industrial centrifugal compressor has been tested in the experimental test rig of the Department of Industrial Engineering (DIEF) of Università degli Studi di Firenze [18]. The stall investigation has been carried out both using piezoresistive dynamic pressure sensors at the diffuser's inlet and an ambient microphone positioned close to the model stage. The scopes of the study were: 1) to check if a simple ambient microphone (i.e. a technology easily applicable also in industrial machines) is able to correctly detect the stall onset; 2) to compare the way the two methods detect the phenomenon; 3) to evaluate the amount of information on the stall features that one could obtain from the two methods.

\section{EXPERIMENTS}

\subsection{Test rig}

The test rig, located at the LINEA laboratory of the Department of Industrial Engineering (DIEF) of Università degli Studi di Firenze, was designed and realized with the aim to perform advanced thermo-fluid dynamic analyses on centrifugal compressors for oil and gas applications [18]. It is installed inside a dedicated test cell realized with soundadsorbing wall both to limit the acoustic noise and to ensure the mechanical containment during the tests. Fig. displays a sketch of the rig. The rig is made of an electric motor driven by an inverter to vary the compressor speed; a flange torque meter for the measurement of torque and power adsorbed by the compressor; the main shaft, which has the twofold scope of supporting and transmitting the rotary motion to the impeller. Finally, on the right end of the rig, the compressor stage and the suction line are installed. This test rig differentiates from industrial heavy-duty ones mainly by the following features: 1) in its basic configuration (Fig. 1) the stage operates with free discharge at the impeller exit; 2) rotor shaft is installed on not lubricated, low friction and high precision bearings. The choice of an open loop configuration allowed for a high flexibility of the rig, in which the assembly/disassembly of different configuration is extremely simple, as well as the installation of a variety of sensors. The adoption of a shaft installed on low friction, high precision, not lubricated bearings, on the other hand, ensured a high radial precision of the shaft-stator coupling and consequently tight coupling tolerances between impeller and related flow path; low rotational frictions ensure minimizing the power adsorbed between 
the torquemeter and the impeller so that the measured torque/power can be associated almost entirely to the compressor fluid dynamic work; furthermore, the absence of a lubricating system makes the rig free of both the lubricating line and the oil control unit with benefits in terms of ease of use, flexibility and cleaning of the rig. Finally, this feature, coupled with a dedicated rotordynamic design, allows the rig to operate safely within the sub-critical range. All the mechanical apparatus is installed on a reinforced-concrete platform placed on 5 rubber buffers to both adsorb mechanical vibrations and to avoid the interaction of the rig with the floor during its functioning.

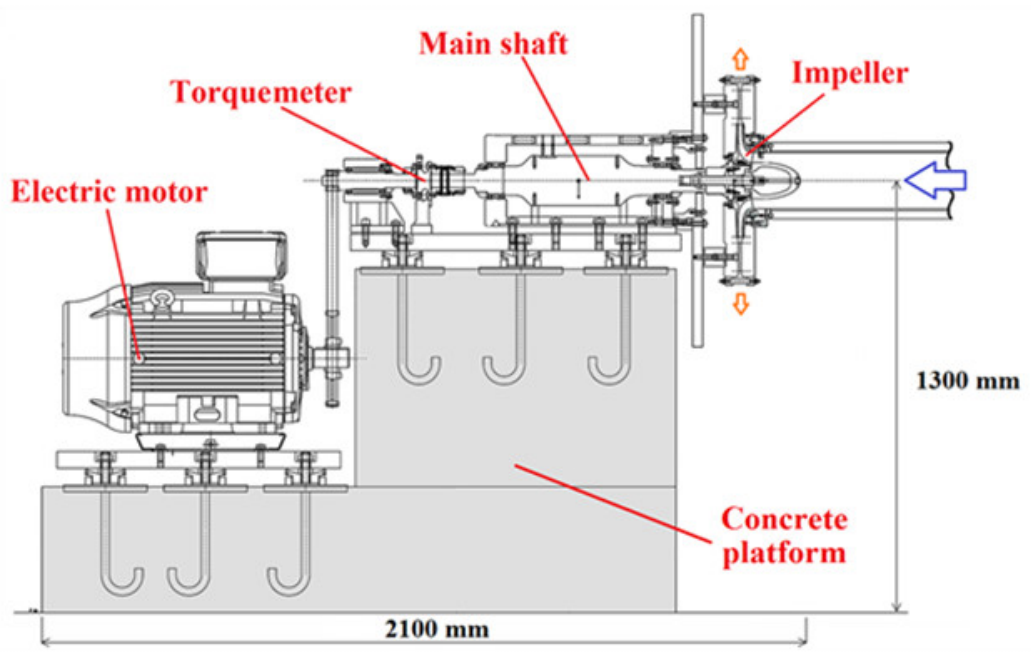

Fig.1. The UNIFI test rig for centrifugal compressors [18].

The cell (Fig.2a) hosting the test rig is provided with an air-exchange system aimed at both cooling down the cell during the tests and balancing the pressure level within the cell itself after the air compressed by the centrifugal compressor is injected. The system design was based on a CFD analysis [18] of the air-exchange process of the entire cell (Fig. 2b), paying attention to avoid undesired fluid-dynamic interactions with the discharge flow out of the compressor stage.
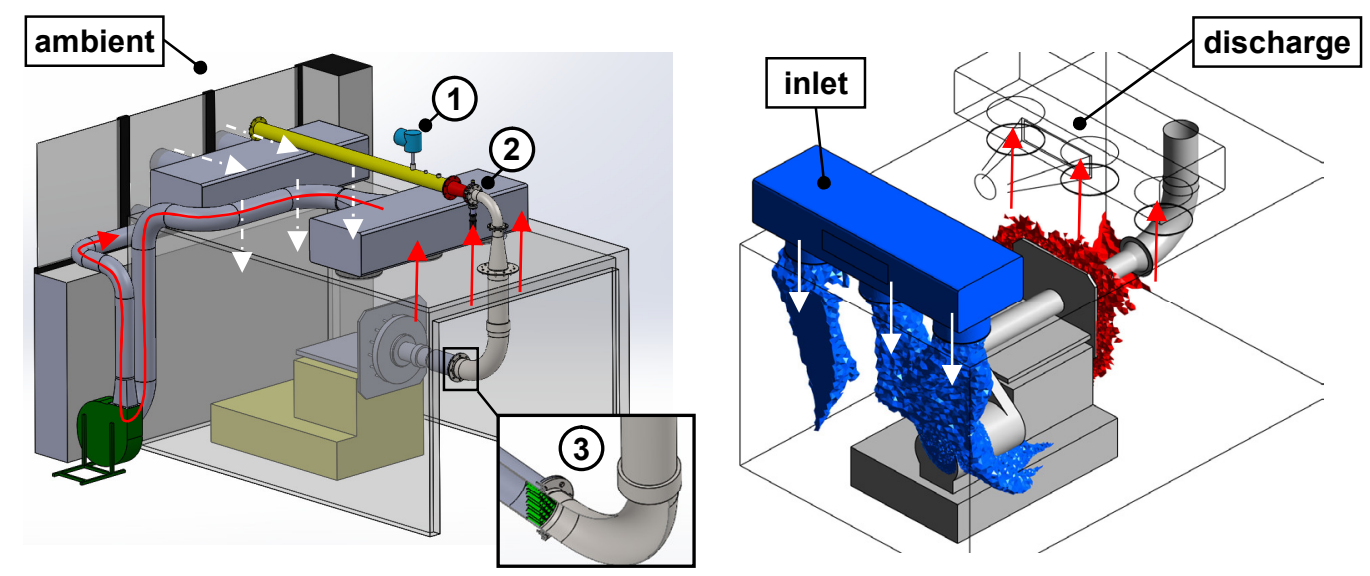

Fig.2. (a) Test cell layout. (b) CFD analysis of the air-exchange system. 
Upstream the impeller, the suction line is composed by: (1) hot wire flowmeter; (2) throttle valve for the control of the inlet air flow. The valve is electro-actuated and allows $0.5^{\circ}$ of opening resolution; (3) a honeycomb rectifier with a length of $75 \mathrm{~mm}$ and a diameter of $200 \mathrm{~mm}$ to straighten the flow and abate swirling components.

\subsection{Model stage}

The compressor stage Fig.3 (a) is composed by: left diaphragm (hub side) (1) coupled with the support plate (2) by means of 12 studs; the impeller (3), which is installed on a special hub (4) and coupled with the rotor (5) by means of a tie rod (6) and related nut; on the right side of the impeller, the ogive (7) is attached; the stage is finally enclosed by the right diaphragm (8). The left and right diaphragms are coupled together using spacers (9) placed on the external diameter of the stage. Back flow phenomena are limited by using two static sealing, the first (10) at the hub side and the second (11) at the impeller inlet; the upper limit of the flow path is limited by an annular spacer (12) that acts like the junction between the intake duct and the compressor stage. Geometrical details of the impeller used in this study cannot be disclosed due to a dedicated agreement with the industrial partner. However, it was a $390 \mathrm{~mm}$ diameter impeller for oil\&gas applications featuring 24 bidimensional blades and having a diffuser width to diameter ratio of 0.021 . Measurements sections are depicted in Fig.3 (b), while relative instrumentation is reported in Table1.
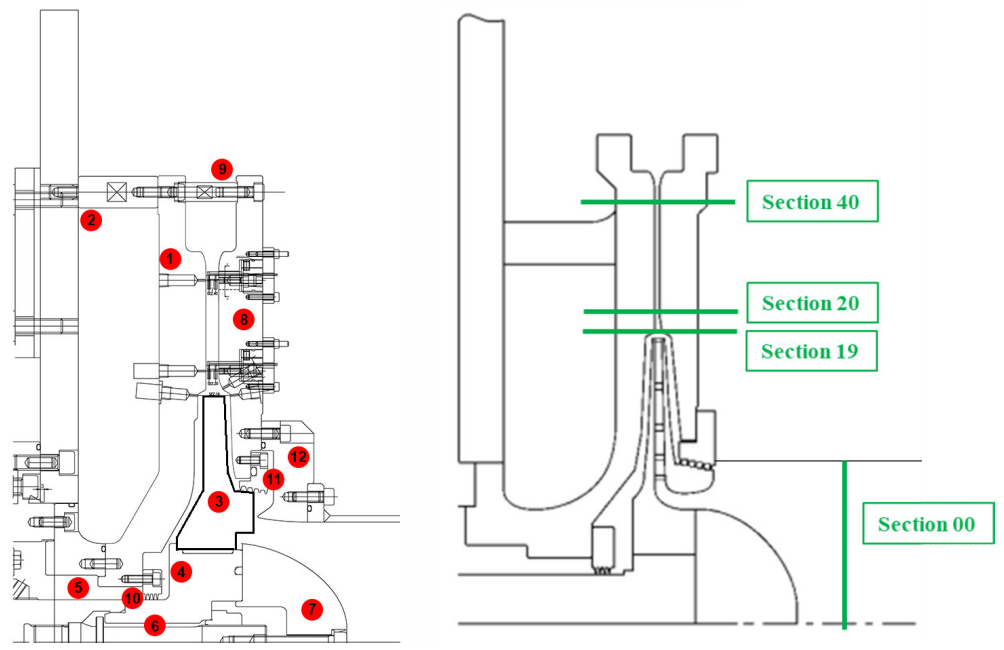

Fig.3. (a) Compressor stage cross-section (impeller geometry is purely qualitative). (b) Measuring sections.

Table1. Instrumentation: $\mathrm{PT}=\mathrm{Total}$ pressure probe, $\mathrm{TT}=$ total temperature probe, $3 \mathrm{H}=3$-hole probe.

\begin{tabular}{|l|l|l|}
\hline Section 00 & Section 20 & Section 40 \\
\hline 4 rakes PT 4 MP & 4 rakes PT 2 MP & 4 rakes PT 2 MP \\
\hline 4 rakes TT 4 MP & 4 rakes TT 2 MP & 4 rakes TT 2 MP \\
\hline 4 static pressure MP & 4 rakes 3H 2 MP & 4 rakes 3H 2 MP \\
\hline$/$ & 2 traversing PT\&3H & 2 traversing PT\&3H \\
\hline$/$ & 8 static pres. MP (4H/4S) & 8 static pres. MP (4H/4S) \\
\hline$/$ & 3 dynamic pressure MP & \multicolumn{1}{c}{} \\
\hline
\end{tabular}


In Fig.4, a scheme of the probes' installation is reported. Total pressure probes allow an incidence angle of $\pm 20 \mathrm{deg}$ and are installed to evaluate the pressure recovery from impeller inlet to diffuser discharge. Total temperature sensors, realized with J-type thermocouples, are used for the evaluation of performance parameters such as efficiency, work coefficient, etc. 3-hole probes are used for the estimation of the yaw angle both at the impeller outlet and at the diffuser outlet in order to characterize the flow trajectory within the diffuser; probes were calibrated in the range of $\pm 30^{\circ}$.

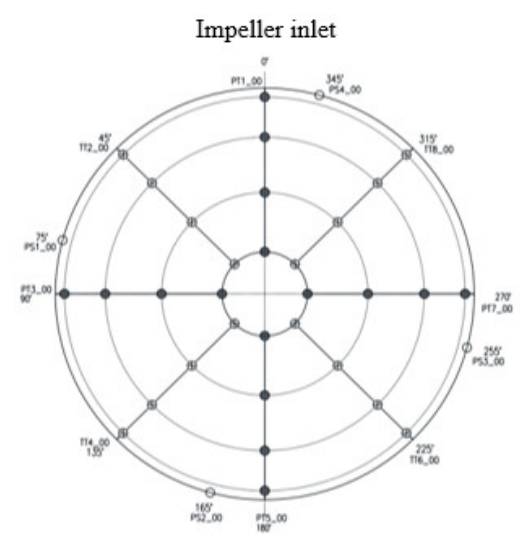

(a)

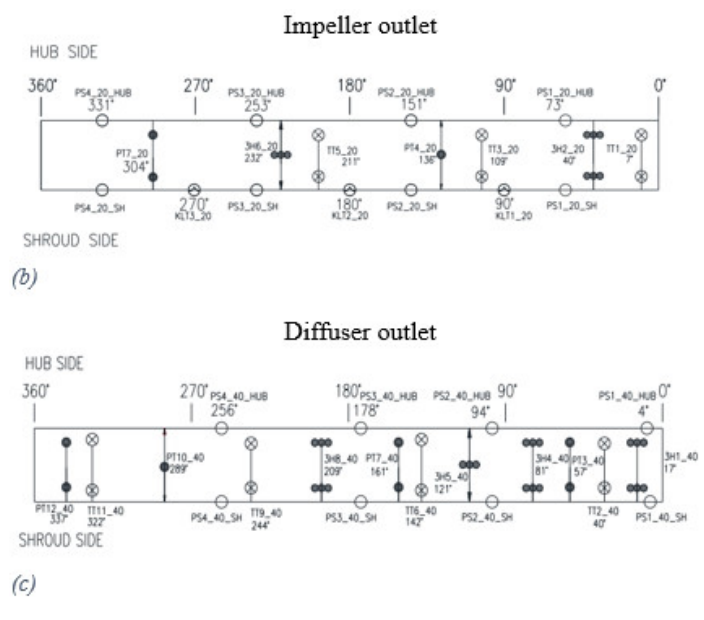

Fig.4. Compressor stage instrumentation: impeller inlet (sec.00), impeller outlet (sec.20) and diffuser outlet (sec.40).

In addition to the discussed conventional measurements, advanced dynamic pressure sensors are flash mounted at the diffuser's inlet and outlet, in order to allow for a dedicated study of unsteady phenomena like the rotating stall in the diffuser subject of the present study. Figure 5 displays a typical sensors' pattern.

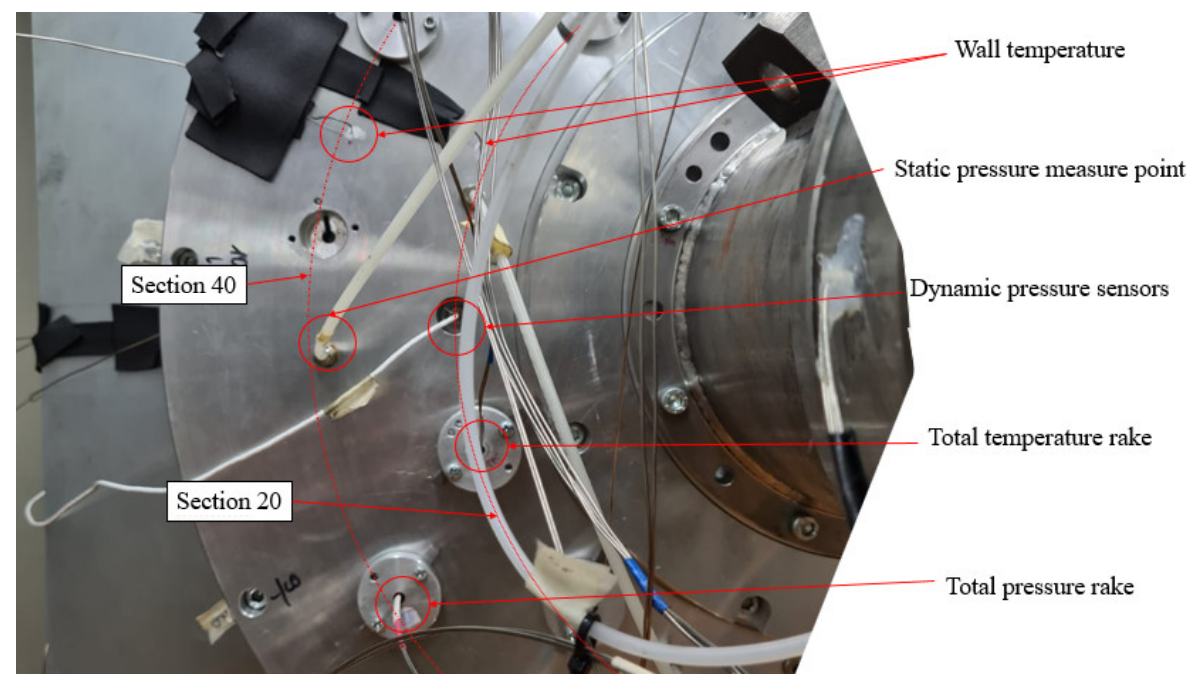

Fig.5. Sensors installation on compressor stage at sections 20 and 40. 
In addition to total temperature, total pressure, three-hole and static pressure probes, two J-type thermocouples are placed at section 20 and 40 in order to estimate the diaphragm wall temperature, and another $\mathrm{J}$ type thermocouple is used for the measurement of cell thermal state. In order to minimize the influence of the thermal exchange along wall, thermocouples were insulated with neoprene and the measuring point, hot junction, was installed in a dedicated blind hole of $1 \mathrm{~mm}$ diameter and $1.5 \mathrm{~mm}$ depth with thermal paste. By doing so, a first guess of the thermal exchange between the stage and the external ambient during the tests can be made.

\subsection{Instrumentation}

For sick of brevity, a synthetic overview of the instrumentation adopted is listed below (and depicted in Fig.6).

- Flow meter: it is installed on the intake line (Fig.2a)), upstream the throttle valve. Since it is a hot wire typology, it was installed respecting the manufacturer specification of a distance equal to 15 diameters from the intake and 5 diameters far from downstream curve. Manufacturer Hendress Hauser, Model T mass 300L, measuring range 0.17 $2.14 \mathrm{~kg} / \mathrm{s}$, accuracy $1 \%$ measured value, output $4-20 \mathrm{~mA}$.

- Torque meter: it is flange typology installed between the electric motor and the compressor shaft by means of a specific flexible junction (Fig.1). Manufacturer $\boldsymbol{H B M}$, model $\boldsymbol{T 4 0 B}$, measuring range $0-100 \mathrm{Nm}$, accuracy $0.03 \%$ of the full-scale.

- Rotating speed meter: the compressor is provided with a 60 teeth phonic wheel integrated on the shaft; the rotational speed is monitored using a hall effect senor. Manufacturer Jaquet, model F12A, output NPN 24V.

- Accelerometers: they are installed one the left side, one on the center and one on the right side of the compressor stator (or cartridge) containing the shaft. They are 3-axial piezoelectric accelerometers. Manufacturer Kistler, model 8763B, measuring range $\pm 50 \mathrm{~g}$.

- Pressure scanners: they are used to measure the steady pressure of PT and $3 \mathrm{H}$ rakes, and the static wall pressures. Given the presence of different pressure levels from impeller inlet to diffuser outlet, scanners with ranges of 1, 2.5, 5, 10 and 30 PSI are used. Manufacturer Scanivalve, model 3217/3218, overall uncertainty $0.02 \%$ FSO, 16 channels.

- Temperature Scanner: it allows to acquire the temperature signals of both rakes of TT and wall temperature of the diffuser. It is provided with an internal cold junction reference while temperatures are acquired in voltage. Manufacturer Agilent, model $34980 A$.

The test bench is provided with an acquisition system based on NI platform and managed by CECO software developed at DIEF in LabView ${ }^{\mathrm{TM}}$ ambient. Data acquisition and control are entrusted to a PXIe 1082 and a cRIO 9027. CECO software is developed on PIXe 1082 which communicates with cRIO 9027 and the other sub-acquisition system as shown in Fig.7. Moreover, PIXe 1082 allows the direct acquisition of high frequency signals such as dynamic pressure sensors for stall onset investigation. CECO SW absolves to the functions of data acquisition, of both steady-state and dynamic phenomena, and control, such as management of rotating speed and throttle valve opening. Also, a first level of safety management is entrusted to CECO SW, that is the control of maximum rotating speed, maximum operating temperatures and admissible accelerations, in case of exceeding the limits the rig automatically shuts down. 

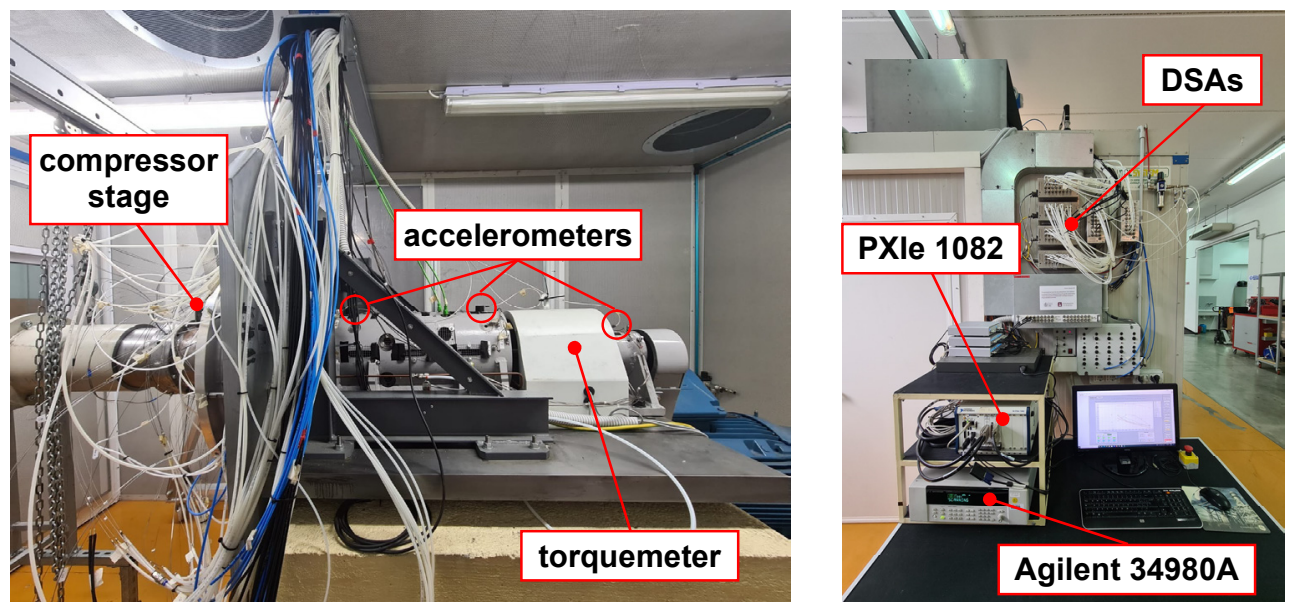

Fig.6 Test bench (a) instrumentation and (b) control system.

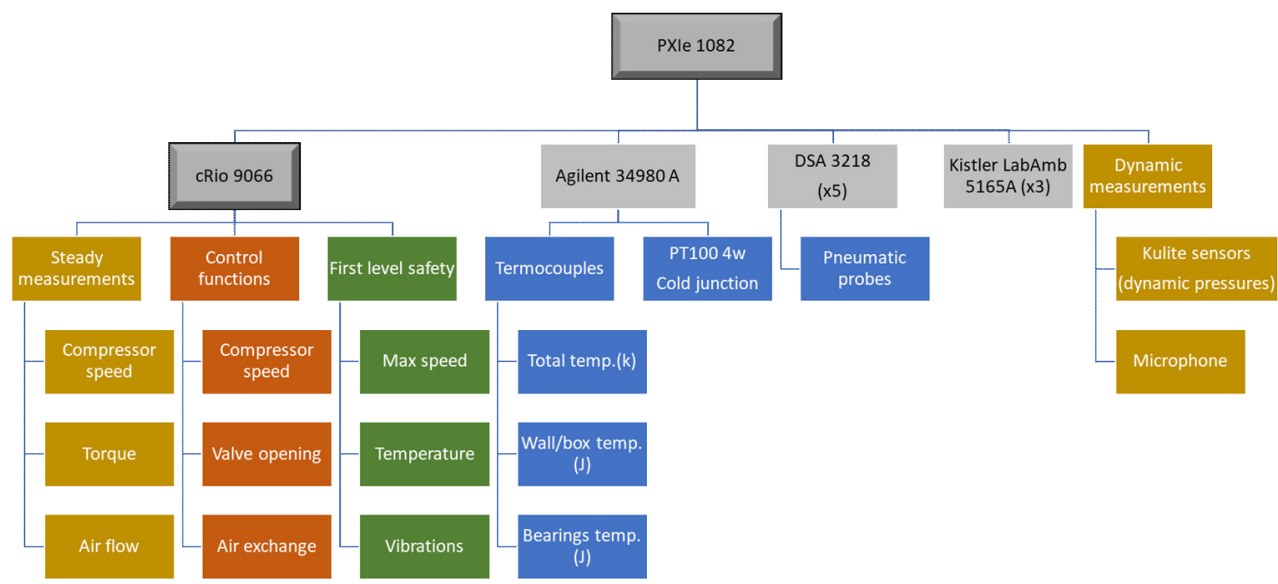

Fig.7. Data acquisition and Control system diagram.

All the acquisition equipment is located outside the test cell in order to preserve it from thermal stress.

Finally, for the scopes of the present study, an ambient microphone is installed inside the compressor cell on a dedicated adjustable support in order to avoid any mechanical interaction with the compressor (Fig.8). The microphone was placed at a $1 \mathrm{~m}$ distance from the diffuser outlet, $1.20 \mathrm{~m}$ height and with an inclination of $45^{\circ}$. The microphone used during the tests is a piezoelectric type one, with the following features:

- $\quad$ Model AVM MI17

- $\quad 1 / 4^{\prime \prime}$ IEPE Measurement Mic

- Frequency response $20 \mathrm{~Hz}-20 \mathrm{kHz}$

- Sensitivity $50 \mathrm{mV} / \mathrm{Pa}$

- $\quad$ Measuring range $26 \mathrm{~dB}$ (A-weighted)

- $\quad$ SPL Peak (5\% Dist.) $130 \mathrm{~dB}$ 
The microphone was acquired by means of a NI9234C series module design for sound and vibration applications; this system allows the acquisition of IEPE sensors with a maximum sample rate of $51.2 \mathrm{kS} / \mathrm{s}$ per channel and $\pm 5 \mathrm{~V}$. The acquisition of the microphone was entrusted to the PXIe 1082 and both the microphone and dynamic pressure sensors signals were simultaneously acquired in order to have a direct comparison between the two different approaches.

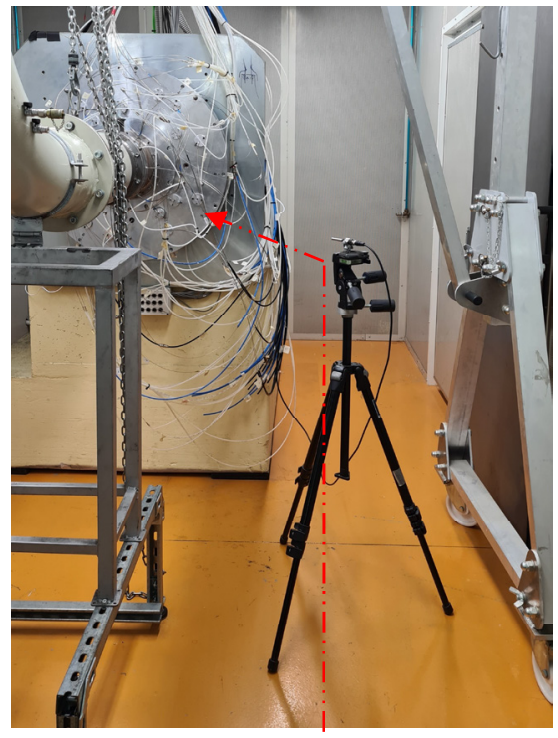

Fig.8. Microphone installation within the test rig cell.

\section{RESULTS}

Figure 9 compares the joint time-frequency graphs in the subsynchronous region for the same test at a peripheral Mach Number $(\mathrm{Mu})$ equal to 0.5 using either a dynamic pressure probe (left) and the microphone (right). While time is advancing, the flow coefficient is progressively reduced. The color scale reports the amplitude of the frequency components in Pascal. Upon examination of the same, some interesting remarks can be done:

- for sufficiently high flow coefficients, the power spectrum of the pressure probe is very clean. The revolution frequency $\left(\mathrm{f}_{1 \times R E V}\right)$ is barely seeable (an artificial black line has been added to help the reader localizing it), testifying a high degree of uniformity between the impeller's passages.

- on the other hand, the power spectrum of the microphone is more complex. The $f_{1 \times R E V}$ is clearly distinguishable (probably due to a singularity not connected with the flow), as well as the contributions of two additional frequencies, approximately around $0.11 \mathrm{f}_{1 \times \mathrm{xEV}}$ (F1) and $0.22 \mathrm{f}_{1 \times R E V}(\mathrm{~F} 2)$, respectively.

- at the same instant in time (approximately at 20s from the acquisition start), both sensors highlight the onset of a further contribution of small amplitude around $0.35 f_{1 \times R E V}(\mathrm{~F} 3)$, progressively increasing.

- then, at $23 \mathrm{~s}$, the onset of a new pattern (that will be revealed to represent the rotating stall) at approximately $0.19 \mathrm{f}_{1 \times R E V}$ arises $\left(f_{\text {stall }}\right)$, coupled with its first harmonics $\left(0.38 \mathrm{f}_{1 \times R E V}\right)$. In case of the pressure sensors, the amplitude of the harmonics is higher than that of the base frequency while the opposite is seen in the microphone. 


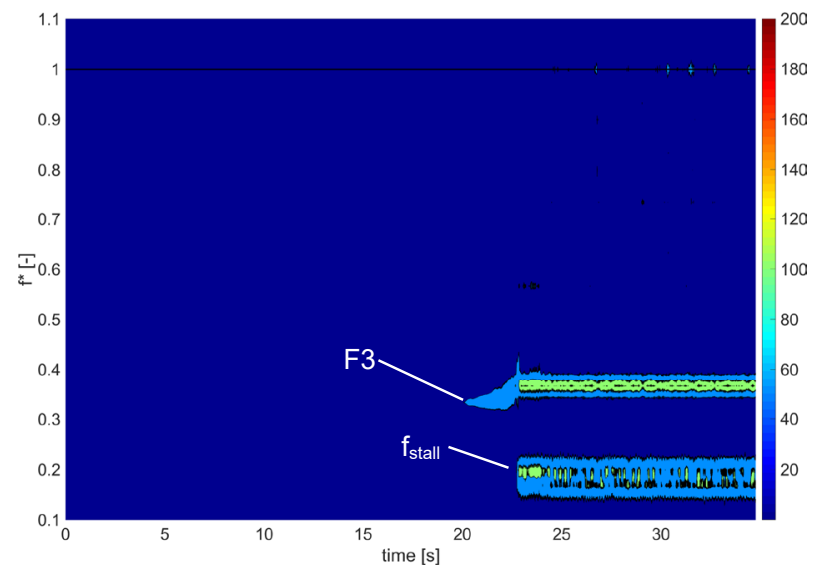

(a)

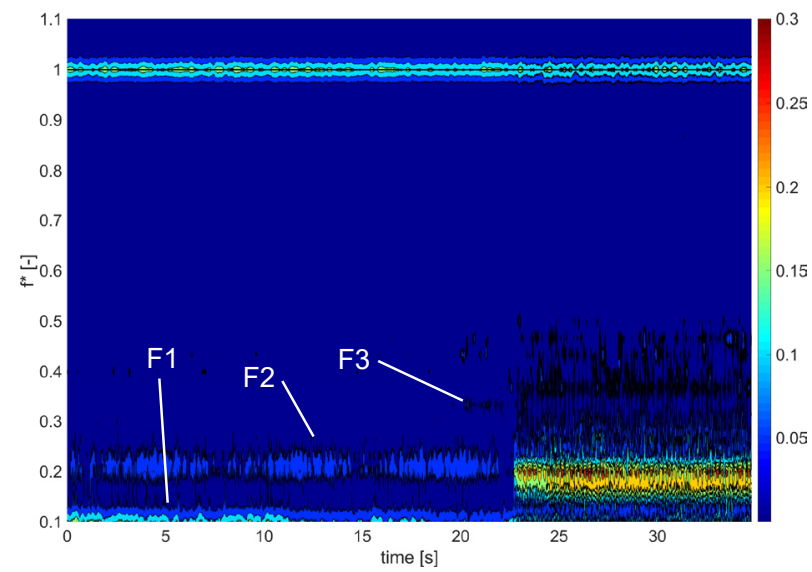

(b)

Fig.9. Joint time-frequency graph of the stall analysis $@ \mathrm{Mu}=0.5$ using either a dynamic pressure sensor (a) or the microphone (b).

To get a better understanding of the phenomenon, the approach presented by [8] was applied to the two dynamic pressure probes available at the impeller exit. Figure 10(a) reports the power spectrum of the signal in a given time instant (i.e. a vertical section of Fig.9a), confirming the pattern already analyzed so far. Then, the signal of one probe has been selected and the autocorrelation function was applied to it; the procedure confirmed that the base frequency (i.e. those maximizing the autocorrelation) was $25 \mathrm{~Hz}$. Then, the autocorrelation function applied to the two sensors, gave a phase difference between then corresponding, if shifted from time to space using that frequency, exactly to the physical phase shift between the sensors. As a result, a single lobe structure was confirmed, having the shape reported in Figure 10b. If one relates these results with those displayed by Figure 9(b), it can be noticed that the microphone correctly only highlighted the stall frequency as that having the stronger amplitude, while the pressure probe sensed the first harmonic as that having the stronger amplitude. This can be probably due to the bi-cusp form of the stall, which is typical of patterns not completely rigid during their revolution [7]. Notwithstanding this, the procedure of [8] was able to correctly reconstruct the pattern anyway. 
In addition, the stall onset parameter ( 7 ) proposed by Bianchini [2] (Eq. 1) was measured for both sensors.

$$
\lambda=\frac{\int_{0}^{R E V-\delta f} P_{t} d f-\int_{0}^{R E V-\delta f} P_{0} d f}{\int_{B P F-\delta f}^{B P F+\delta f} P_{0} d f}
$$

At each specific instant in time $t$, the parameter correlates the area subtended to the power spectrum $(P)$ of a dynamic pressure signal before the revolution frequency (excluded) to the intensity on the blade passing frequency $(B P F)$ peak, again analyzed in term of area subtended to the peak rather than of peak amplitude ( $d f$ is the frequency resolution). This dimensionless number was originally developed only for piezoelectric dynamic pressure sensors, and it was then of particular interest to test it also with piezoresistive-type sensors and also with the microphone. In case of the piezoresistive sensors (Fig.11a), the original observations of Bianchini et al. seem to be confirmed totally, with the sampi parameter reaching the value of 1 as soon as the stall pattern is established. If, on the one hand, this could be somehow expected, since the type of sensor was not deemed to modify the physics of the problem, it was very interesting to have this confirmation also in case of a different stage type with the diffuser discharging freely in the environment. On the other hand, it was even more interesting to note that also if the number was calculated on the microphone, it was able to nearly reach the same unit value at the stall onset. This was absolutely not trivial to be expected since the range of phenomena sensed by the tow different measure is different. Anyhow, this seems to suggest that the physics proposed by the stall onset parameter of Bianchini (i.e. the relation between the amplitude of all the subsynchronous components and that of the blade passing frequency) are strongly linked to the real onset mechanism of the stall pattern.

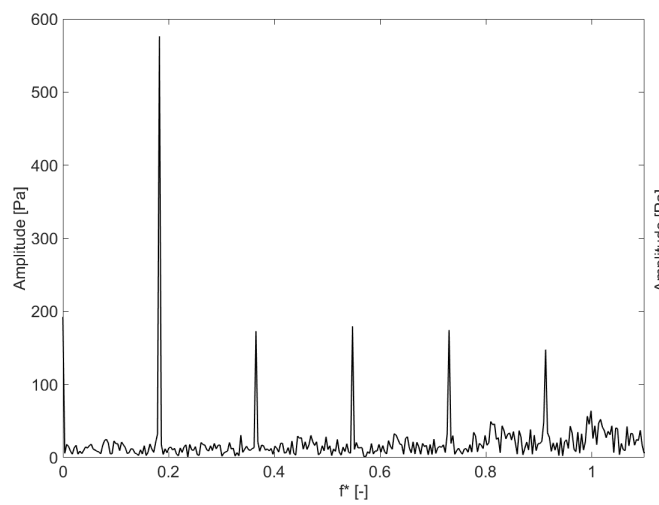

(a)

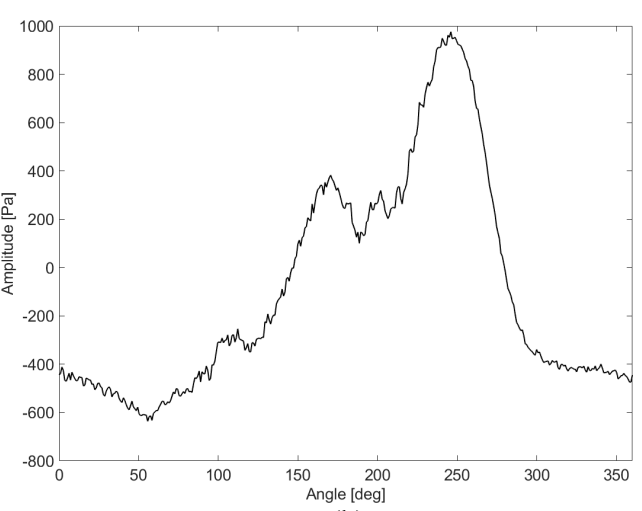

(b)

Fig.10. (a) Power spectrum of a dynamic pressure sensors in the subsynchronous frequency range during stall conditions @ $\mathrm{Mu}=0.5$; (b) Spatial reconstruction of the stall pattern. 


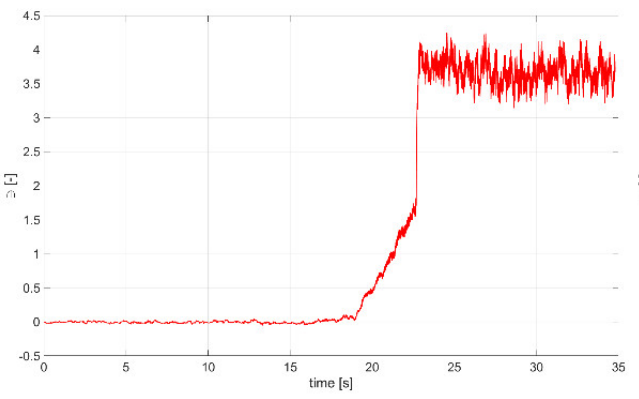

(a)

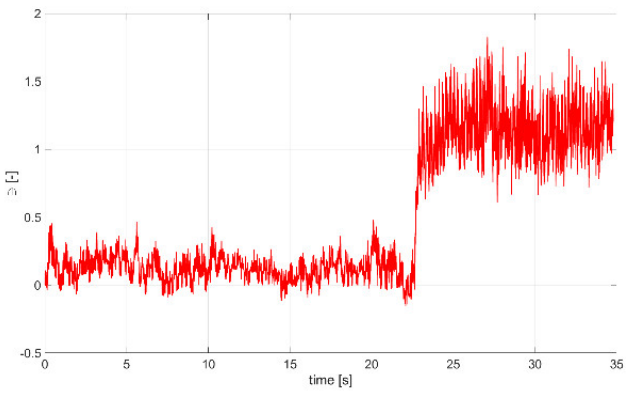

(b)

Fig.11. Trends of the stall onset parameter $\rightarrow @ \mathrm{Mu}=0.5$ using either a dynamic pressure sensor (a) or the microphone (b).

Similar considerations can be also repeated for the case at $\mathrm{Mu}=0.7$ (Fig.12). In this case, the power spectrum in stall conditions is unusually dirtier, whit multiple frequency components seeable.

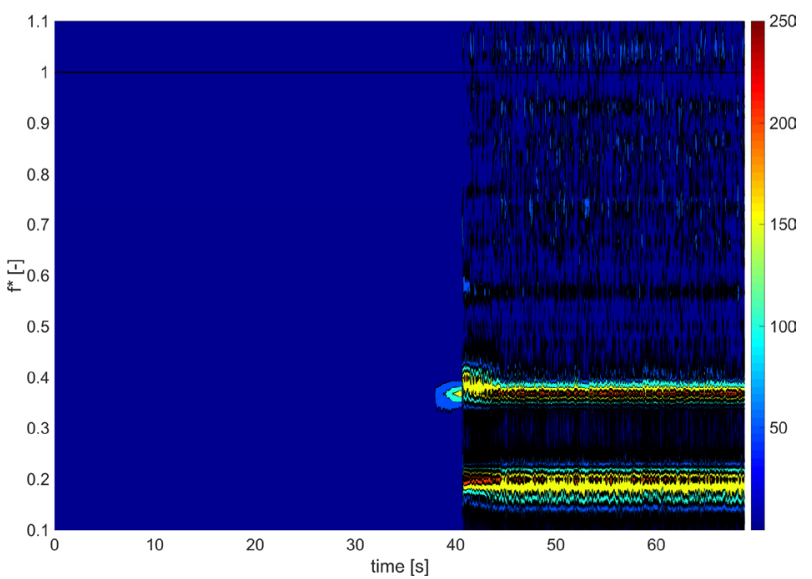

(a)

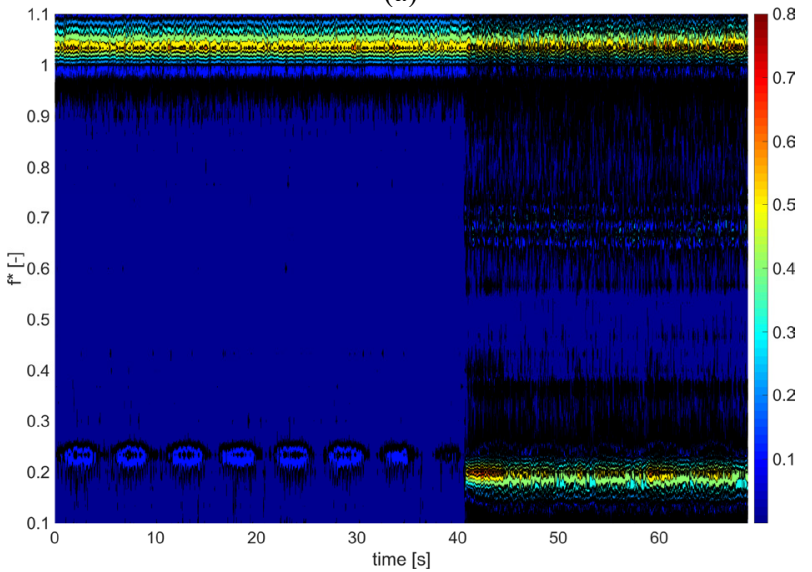

(b)

Fig.12. Joint time-frequency graph of the stall analysis $@ \mathrm{Mu}=0.7$ using either a dynamic pressure sensor (a) or the microphone (b). 
The reasons for this are not completely understood, and probably due to the acquisition system, even though several replications of the same test confirmed that the physics of the problem were consistently reproduced. As an additional effect, the stall onset parameter (Fig.13) highlighted in both cases a threshold slightly higher than that seen before (approximately at 2), even if it was also apparent that the stall onset was now very abrupt, with a steep variation of the parameter.

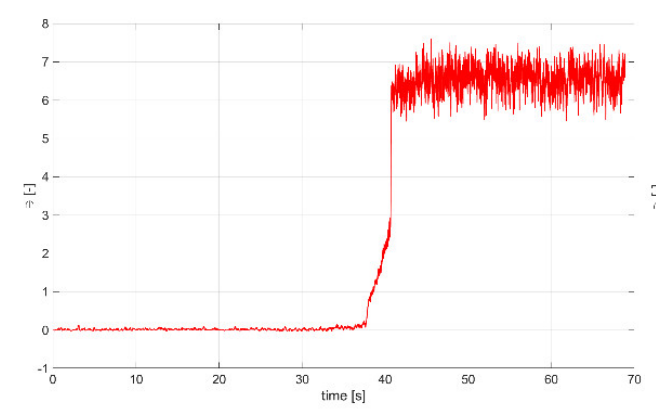

(a)

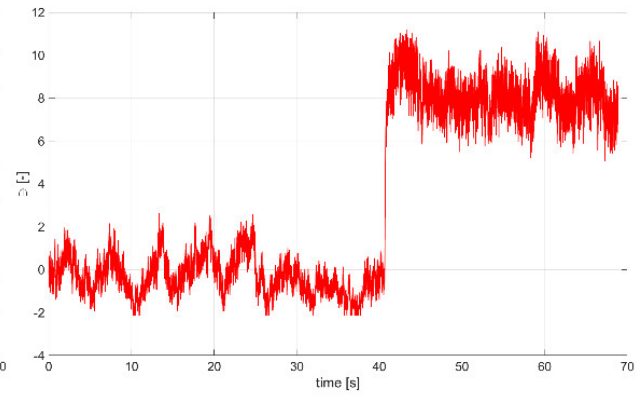

(b)

Fig.13. Trends of the stall onset parameter $\rightarrow @ M u=0.7$ using either a dynamic pressure sensor (a) or the microphone (b).

\section{CONCLUSIONS}

In the study, experimental tests have been carried out in a dedicated experimental test rig to compare the capabilities of two different methods to detect the onset of vaneless diffuser rotating stall in a centrifugal compressor, i.e. dynamic pressure sensors installed in the diffuser and an ambient microphone.

Results showed that the ambient microphone was able to detect the onset of rotating stall correctly and promptly. Moreover, the analysis of its power spectrum revealed that the relationship between the amplitude of the subsynchronous region and that of the blade passing frequency theorized in case of dynamic pressure sensors through the dimensionless stall onset parameter $\rightarrow$ is still valid. Although additional tests are required, also in case of stage configurations including a volute, this seems to suggest that the use of ambient acoustic measurements could find room for automatic detection of rotating stall onset in the near future. On the other hand, it should be remembered that this kind of measurement does not provide any relevant information about the characteristics of the stall pattern, and hence on the destabilizing force connected to it; in this view, it cannot be implemented into an automatic method to control the rotordynamic response of the compressor. Future work will be also devoted at understanding the validity of the approach in case of real industrial installations, where spurious noise sources could alter the measurement of the microphone. In this view, while a correct mounting of the same as close as possible to the diffuser could help limiting environmental noise, one should anyhow remember that rotating stall is characterized by a very strong noise (very often much higher that other environmental sources) and with a very specific frequency. It is then believed that the proposed approach could work properly also in case of real installations.

Thanks are due to Baker Hughes for supporting the research program on rotating stall at Università degli Studi di Firenze and for providing the permission of showing experimental data. 


\section{References}

1. NREL, (2014)

2. A. Bianchini, G. Andreini, L. Ferrari, D. T. Rubino, and G. Ferrara, Journal of Engineering for Gas Turbines and Power 141, (2019)

3. Y. Senoo, Y. Kinoshita, and M. Ishida, Journal of Fluids Engineering, Transactions of the ASME 99, 104 (1977)

4. P. Frigne and R. Van Den Braembussche, in (1982), pp. 365-381

5. G. Ferrara, L. Ferrari, and L. Baldassarre, The International Journal of Rotating Machinery 10, 433 (2004)

6. B. F. Evans and A. J. Smalley, (1984)

7. A. Bianchini, D. Biliotti, M. Giachi, E. Belardini, L. Tapinassi, L. Ferrari, and G. Ferrara, in (2014)

8. A. Bianchini, D. Biliotti, G. Ferrara, L. Ferrari, E. Belardini, M. Giachi, L. Tapinassi, and G. Vannini, Journal of Engineering for Gas Turbines and Power 135, (2013)

9. J. M. ||Marshall Sorokes, (2000)

10. D. Biliotti, A. Bianchini, G. Vannini, E. Belardini, M. Giachi, L. Tapinassi, L. Ferrari, and G. Ferrara, Journal of Turbomachinery 137, 1 (2015)

11. D. E. Bently and P. Goldman, Orbit First Quarter 2000, 32 (2000)

12. I. G. Khisameev, Ya. Z. Guzel'baev, and A. L. Khavkin, Chemical and Petroleum Engineering 43, 537 (2007)

13. D. R. Smith and J. C. Wachel, Turbomachinery International 24, 21 (1983)

14. L. Ishimoto, (2012)

15. P. B. Lawless and S. Fleeter, Journal of Turbomachinery 117, 87 (1995)

16. A. Bianchini, D. Biliotti, D. T. Rubino, L. Ferrari, and G. Ferrara, Journal of Turbomachinery 137, 1 (2015)

17. N. Aretakis, K. Mathioudakis, M. Kefalakis, and K. Papailiou, Journal of Engineering for Gas Turbines and Power 126, 840 (2004)

18. A. Bianchini, L. Ferrari, and G. Ferrara, in (2016) 